\title{
EODC: An Energy Optimized Dynamic Clustering Protocol for Wireless Sensor Networks using PSO Approach
}

\author{
C. Jothikumar, R. Venkataraman
}

\author{
C. Jothikumar* \\ Department of Computer Science \\ SRM Institute of Science and Technology \\ Chennai, India \\ *Corresponding author: jothikumar.c@ktr.srmuniv.ac.in
}

\author{
Revathi Venkataraman \\ Department of Computer Science \\ SRM Institute of Science and Technology \\ Chennai, India \\ revathi.n@ktr.srmuniv.ac.in
}

\begin{abstract}
A Wireless Sensor Network comprises many small wireless nodes that helps to sense, gather, process and communicate. One of its primary concerns is to optimize the consumption of energy and extend the network lifespan. The sensor nodes can be clustered to increase its lifespan further and this can be accomplished by choosing cluster head for every cluster and by performing data fusion on the cluster head $(\mathrm{CH})$. The proposed system is using an energy efficient hierarchical routing protocol named Energy Optimized Dynamic Clustering (EODC) for clustering large ad-hoc WSNs and direct the data to reach the sink. The collected data is received by the sink from the set of cluster heads after every round. The cluster head is selected using the Particle Swarm Optimization (PSO) approach; the allocation of cluster members is based on the Manhattan distance. The metrics used to find the fitness function are location, link quality, energy of the active and inactive nodes. The system employs the shortest path approach to communicate between the cluster heads till it reaches the base station. By this, the energy efficiency and network lifetime have been increased. The analysis and outcomes show that the EODC was found to outperform the existing protocol which compares with this algorithm.

Keywords: Clustering, Wireless Sensor Network (WSN), energy efficiency, routing, particle swarm optimization (PSO).
\end{abstract}

\section{Introduction}

In recent times, Wireless Sensor Networks (WSN) are procuring research attention. The nodes communicate with their neighbors to transmit the data till it reaches the destination $[2,3]$. The potential utilization of sensor networks is in various fields such as target monitoring, environment tracking, healthcare and industries. A WSN generally consists of sensor nodes, used for sensing, processing and communicating to reach the sink. These small sized sensor nodes make it easier to collect the data about the environment. A WSN has various characteristics, including less battery life, power constraints, coping with node failures, and dynamic network topology, etc.

Flooding is a flat based routing protocol which allows to flood the sensed data in the network through the neighbor nodes $[8-10,12]$. Since the data are flooded throughout the network, the system drains due to duplication of data, overlapping and resource blindness. In direct diffusion the sink initiates the request to the source nodes. The source node saves the path of the sink, once the message is received. The routing consists of interest propagation and gradient setup. Interest propagation refers to the query from the sink where the gradient setup transmits the data 
to reach the sink. An adaptive routing protocol called as the Sensor Protocol for Information via Navigation (SPIN) assigns high-level name to describe the data. The SPIN performs better by saving energy than the flooding approach. LEACH makes use a stochastic algorithm for selection of $\mathrm{CH}$. The data gathered from the individual cluster member is fused by the $\mathrm{CH}$ and forwarded to reach the sink through single-hop communication. To avoid interference between the clusters while transmitting the data, a direct sequence spread spectrum is used. The $\mathrm{CH}$ is randomly chosen from the available nodes in the respective cluster to ensure that all the nodes should become a cluster head once. This algorithm can hence reduce the volume of data forwarded directly to a base station (BS). These cluster heads are unevenly distributed in the network. If they happen to be selected randomly only from a dense area, this may lead to increased energy consumption during data transmission. Since the CHs are elected based on probability, it may lead to overhead and increase in the network load and it is not scalable. The level of energy consumed is a prime concern in draining the network. In order to improve the efficiency, we developed an Energy Optimized Dynamic Clustering (EODC) protocol that uses location, link quality, energy of active and inactive nodes to find the fitness function for selecting the $\mathrm{CH}$. The proposed system reduces the energy consumption by selecting the optimized $\mathrm{CH}$ and routes the packet through the near optimal path in the network.

The paper is structured as follows. The related work of the system is described in section 2. The Section 3 describes the EODC protocol. The results of the simulation and discussion are shown in section 4 . At last, in section 5 , the conclusion segment is covered.

\section{Related work}

The design of energy efficient routing protocol is such that it improves the performance and network lifespan. In this segment five hierarchical routing protocols are briefly discussed.

In a wireless sensor network, Low-Energy Adaptive Clustering Hierarchy-Centralized (LEACHC) is the effective clustering approach for data communication. For the first round, the clusters are created and the selection of $\mathrm{CH}$ is based on a stochastic algorithm [11]. The BS receives the message about energy as well as the location of nodes. The BS computes the $\mathrm{CH}$ as per the received data. The nodes which have the energy more than the average of that set of nodes are considered as $\mathrm{CHs}$ from the second round. The CHs fuse the data gathered from the individual cluster member and forward it towards the BS. For saving energy, each member uses the time division multiple access (TDMA) scheduling for transmitting data to reach the $\mathrm{CH}$ and once the time is out, the node goes to sleep. The $\mathrm{CH}$ must be active to receive the data continuously and further pass the collected data to the base station. The LEACH-C being a one-hop communication protocol helps in direct transmission from $\mathrm{CHs}$ to BS. The system performs better than the flat based routing approach by employing a clustering technique.

Inside the Base Station Controlled Dynamic Clustering Protocol (BCDCP), the clusters are formed with an even number of nodes to balance the energy level [16, 20,26]. The energy of overall nodes is computed by the BS. The nodes having the energy above the average are chosen as the $\mathrm{CH}$ for the next round. The selections of the $\mathrm{CH}$ are uniformly placed throughout the sensor field to maximize the distance between the $\mathrm{CH}$ in each splitting step. The received data are transmitted to the required $\mathrm{CH}$ using TDMA scheduling. The $\mathrm{CH}$ aggregates the received data before forwarding it. The protocol performs the multi-hop routing technique among the $\mathrm{CHs}$ for data forwarding using the minimum spanning tree approach. A single $\mathrm{CH}$ communicates the information to the BS; the involved $\mathrm{CH}$ communicating the data will have high energy for transmission.

Inside the General Self-Organized Tree-Based Energy-Balanced Routing Protocol (GSTEB), the root node is assigned by the BS depending on the remaining energy and its associated ID 
is broadcasted to every node $[28,29]$. Similarly, the nodes in the sensor region, select their parents based on the energy of the node by coordinating with their neighbor and every child node transmits the data to their parents within the given TDMA slot. The parent node communicates with the neighbor parents in the form of a tree structure till the data reaches the root node. Here, the data is fused and forwarded by the root node to the BS. The root node is dynamic to balance the load in the communication region. The energy of the sensor nodes in each round is known by the BS and this message favors in creating the topology for the round coming next.

The Hybrid Hierarchical Clustering Approach (HHCA) is a clustering hierarchy approach that performs the operation of the dual clustering mechanism with distributed clustering and centralized grid $[14,23,25,27]$. Distributed clustering is a clustering approach where the nodes are distributed randomly and divided into sub clusters and the cluster members pass the data to the cluster head in the sub cluster. The $\mathrm{CH}$ in the sub cluster converses the shared data to the centralized grid. The BS computes the fuzzy C- means approach for the centralized grid and broadcasts this message in the network. If the ID of the node matches with the broadcast ID that node will act as the centralized grid. Distributed clustering uses the LEACH approach for clustering and $\mathrm{CH}$ selection. The cluster members utilize the TDMA schedule to share the data to the sub cluster. The sub cluster (distributed clustering) transmits the data to the centralized cluster (grid) through CSMA or CA mechanism. The centralized grid forwards the data to the sink directly without correlation.

Heuristic Algorithm for Clustering Hierarchy (HACH) is a clustering technique that performs the operation of the clustering mechanism with inactive nodes by selecting the low energy nodes to be in the sleep state based on a stochastic process that should not affect the network coverage $[5,6,13,18,21]$. The maximum coverage effect is employed in the network to select the inactive nodes to maintain the coverage area. The sink computes the Euclidean distance between the nodes and to itself. The computations are based on the coordinates and energy values received by the sink from all the nodes. The $\mathrm{CH}$ selection was broadcast to every node present in the network. If the node ID and the ID received by the node are the same, then that node will be the $\mathrm{CH}$. The election of the $\mathrm{CH}$ deals with a heuristic crossover approach that is spatially distributed in the detecting range, and the selected $\mathrm{CH}$ has high energy. As far as the proposed system is concerned, we consider LEACH-C, HHCA and the $\mathrm{HACH}$ protocol for comparison.

\section{Energy Optimized Dynamic Clustering (EODC) protocol}

\subsection{Network model}

The nodes are thrown randomly into a square region to observe the environment continuously. $N$ represents the total number of nodes, where $N=\left\{n_{1}, n_{2}, \ldots, n_{n}\right\}$. Assumptions carried out in a sensing region are

- The BS is fixed at the top of the sensing region

- All nodes are stationary in the deployment region

- Being homogeneous, the nodes have the same energy

- The adjustment of transmission power is made according to the received signal strength indicator (RSSI) value of the nodes 


\subsection{Energy consumption model}

The energy of the data transit includes transmission circuitry and the volume of the data being transmitted. Similarly, even the energy of reception includes the volume of data reception. The energy consumption equation is given as: The energy required to transmit a unit of data is,

$$
\begin{aligned}
& E_{T r}(m, d)=E_{c}(m)+E_{a m p}(m, d) \\
& \Rightarrow E_{T r}(m, d)=m E_{c}+m \epsilon_{f s} d^{2} ; d<d_{0} \\
& \Rightarrow E_{T r}(m, d)=m E_{c}+m \epsilon_{m p} d^{4} ; d \geq d_{0}
\end{aligned}
$$

The energy required to receive a unit of data is

$$
E_{R r}(l)=E_{c}(m)=m E_{c}
$$

Where, $E_{T r}$ and $E_{R r}$ are the transmission and reception energy respectively, $E_{c}(m)$ is the energy consumption per bit of transceiver circuitry, $\mathrm{m}$ is the message transmitted, and $\mathrm{d}$ indicates the level of distance measured between the nodes. Depending on the transmission range, free space $\left(d^{2}\right)$ or multipath $\left(d^{4}\right)$ propagation is used.

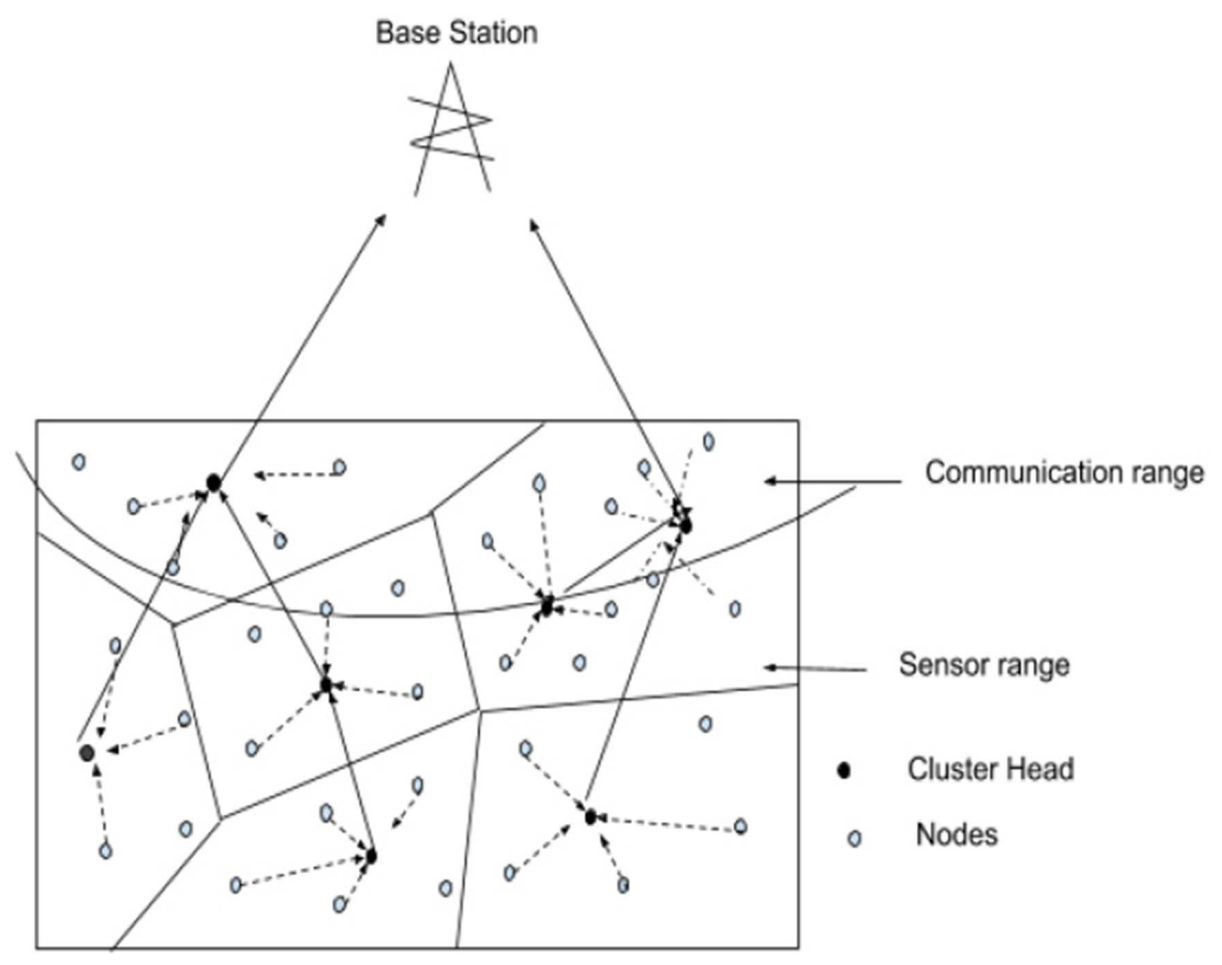

Figure 1: System Architecture of EODC

\subsection{Operation of the EODC algorithm}

In EODC, the nodes form the cluster using the PSO approach. The fitness function used is computed by the $\mathrm{BS}$ for the selection of $\mathrm{CH}$ and the cluster members (CM) are joined using the Manhattan distance. The purpose of the EODC is to reduce energy utilization in clustering 
and data transmission $[1,4,7,15,17,19,22,24,30]$. The system employs the HACH approach for the selection of the inactive nodes in the clusters. The Stochastic Selection of Inactive Nodes (SSIN) protocol introduces the sleep scheduling mechanism to choose inactive cluster members. The coverage effect $\left(C_{e}\right)$ is the effect of identifying the active nodes by putting some nodes to sleep according to the coverage. The number of active nodes present in the network is known which depends on the accumulated coverage effect $\left(A C_{e}\right)$ of all the clusters. The received data are fused by the $\mathrm{CH}$ and forwarded to the nearest $\mathrm{CH}$ to form a multi- hop chain till the data reaches the $\mathrm{BS}$. The $\mathrm{CH}$ in the communication region forwards the data to BS. A shortest path approach was employed to achieve the near optimal path to the BS.

The algorithm consists of two phases, namely:

1. Formation of Cluster and CH Selection using PSO;

2. Data Transmission and Forwarding.

\section{Formation of cluster and $\mathrm{CH}$ selection using PSO}

In the proposed system, the selection of $\mathrm{CH}$ adopting the PSO approach includes the location of the node, link quality between the nodes, and the energy of the active and inactive nodes. The $\mathrm{CH}$ should remain awake always for data reception and transmission. The fitness function of the $\mathrm{CH}$ is calculated using these metrics. $F_{C H}$, fitness function of $\mathrm{CH}$ is given as an optimization technique and is expressed as,

$$
F_{C H}=\gamma \times L O C+(1-\gamma) \times L Q+(1-\gamma) \times E_{n}^{C_{k}}+(1-\gamma) \times E_{m}^{C_{k}}
$$

Location(Loc) gives the identity of the nodes in the network. The computation gives the distance between the neighbor nodes, as well the distance staring from nodes until the $\mathrm{CH}$ of the cluster, $C_{k}$.

$$
L O C=\min _{k=1,2, \ldots, K}\left\{\sum_{n_{i} \in C_{k}} \frac{d\left(n_{i}, C H_{k}\right)}{\left|C_{k}\right|} / \frac{d\left(n_{i}, B S\right)}{\left|C_{k}\right|}\right\}
$$

Where, $d\left(n_{i}, C H_{k}\right)$ gives the distance from $n_{i}$ to $C H_{k}, d\left(n_{i}, B S\right)$ represents the distance from $n_{i}$ to BS, and $\left|C_{k}\right|$ is the length of the cluster

Link Quality (LQ) is the quality of the signal between nodes, ni and the $\mathrm{CH}$ in the cluster. The nodes in the $\mathrm{CH}$ receive the advertisement message. The strength of the signal is known by the nodes in message reception. The RSSI or ETx (Expected Transmission Count) is used to calculate the quality of the link, LQ. Here, ETx is a measure of transmission for the successful delivery of a packet from the sender to the receiver. The computation of paths along the links refers to ETT (expected transmission time) and is inversely proportional to link quality.

Therefore, $\operatorname{RSSI} \alpha \operatorname{ETx} \alpha\left(\frac{1}{E T T}\right)$

$$
L Q=\min _{k=1,2, \ldots, K}\left\{\sum_{n_{i} \in C_{k}} \frac{E T x_{\left(n_{i}, C_{k}\right)}}{\left|C_{k}\right|}\right\}
$$

Where, $\operatorname{ETx} x_{\left(n_{i}, C_{k}\right)}$ is the maximum successful transmission of data from $n_{i}$ to $C_{k}$, and $\left|C_{k}\right|$ is the length of the cluster.

The energy of the active nodes $\left(E_{n}\left(C_{k}\right)\right)$ inside the network is the set of non-CH nodes which address the $\mathrm{CH}$ through TDMA scheduling and the current $\mathrm{CH}$. The energy consumption of the 
cluster head $(\mathrm{ECH})$ includes the transmission energy of the advertisement message and TDMA schedule. The reception energy includes JOINT_REQ message and DATA message. The total energy dissipation of the $\mathrm{CH}$ is represented as,

$$
E_{C H}=E_{T r}\left(C H \_A D V, C H \_T D M A\right)+E_{R r}\left(J O I N T \_R E Q \_C H, D A T A \_C H\right)
$$

Similarly, the energy dissipation of the non $\mathrm{CH}$ (active nodes) belonging to the cluster is represented as:

$$
E_{n o n-C H}=E_{T r}\left(J O I N T \_R E Q \_C H, D A T A_{-} C H\right)+E_{R r}\left(C H \_A D V, C H \_T D M A\right)
$$

The nodes which communicate with the $\mathrm{CH}$ in the current round are called as active nodes. The energy requirement of the active nodes belonging to the network is given as the sum of all the optimum clusters.

$$
E_{n}^{C_{k}}=\sum_{k=1, n_{i} \in C_{k}}^{K} \frac{E\left(n_{i}^{k}-1\right)}{E\left(C H_{k}\right)}
$$

Where, $E\left(C H_{k}\right)$ is the active cluster heads energy.

The energy of the inactive nodes $\left(E_{m}^{C_{k}}\right)$ is the set of nodes in the clusters which remain in sleep mode in the current round and on further iteration, these nodes will wake and another set of nodes become inactive. The energy of the inactive nodes is calculated as,

$$
E_{m}^{C_{k}}=\sum_{k=1, m_{j} \in C_{k}}^{k} \frac{E\left(m_{j}^{k}\right)}{\min E\left(m_{j}^{k}\right)}
$$

Where, $\min \left(E_{m}^{C_{k}}\right)$ is the minimum energy of the inactive nodes.

After computing the metrics, the fitness function that needs to minimize is given as

$$
F_{C H}=0.25 \times L O C+0.25 \times L Q+0.25 \times E_{n}^{C_{k}}+0.25 \times E_{m}^{C_{k}}
$$

Where, $\gamma=0.25$

The fitness function is computed by the BS which broadcasts the message throughout the network. If the node ID matches with the ID of the node sent by the BS, then the node will act as a $\mathrm{CH}$. The energy consumed by the $\mathrm{CH}$ includes the energy of data reception, fusion, and transit. The node will act as $\mathrm{CH}$ till the fitness function value is lesser than the threshold value. The energy of data communication includes the length of the data and energy required for the transmitter circuitry in addition to the energy required for amplification to forward the data through free space propagation, if $d<d o$. The consumption of energy in the $\mathrm{CH}$ for data transmission is given as,

$$
E_{C H}=m E_{c}\left(u_{n}^{C}-1\right)+m E_{D A}\left(u_{n}^{C}\right)+m E_{c}+m \epsilon_{f s} d^{2}
$$

where $u_{n}^{C}$ represents the number of unique nodes in each cluster.

Similarly, the energy of data reception includes the length of the data and energy required for receiver circuitry. The energy consumed by the non-CH for data transmission is given as,

$$
E_{n o n-C H}=m E_{c}+m \epsilon_{f s} d^{2}
$$


The total consumption of energy for the single cluster includes the consumption of energy for the non- $\mathrm{CH}$, of the cluster in intra cluster communication in addition to the energy consumption of the $\mathrm{CH}$ in the given cluster. The total energy consumed by the clusters is calculated as,

$$
E_{\text {clust }}=\left(u_{n}^{C}-1\right) E_{n o n-C H}+E_{C H}
$$

Since, $N$ represents the number of nodes and $M$ indicates the sensor region, the selection of optimal clusters in the system is given using the equation,

$$
K_{\text {opt }}=\frac{\sqrt{N}}{\sqrt{2 n}} \sqrt{\frac{\epsilon_{f s}}{\epsilon_{m p}}} \times \frac{M}{d^{2}}
$$

The energy consumed by the entire network is the product of consumption of energy for the single cluster and the optimal number of clusters, $K_{\text {opt }}$ present in the sensing region. The total energy consumption by the network is given as,

Substituting (9) and (10) in (11)

$$
E_{\text {network }}=K_{\text {opt }} \times E_{\text {clust }}
$$

The assumption is being carried out as uniform deployment of nodes in the detecting region. The distance from the nodes to the $\mathrm{CH}$ is given as

$$
\begin{aligned}
E[d]=\iint \sqrt{x-x_{C H}^{2}+\left(y-y_{C H}\right)^{2}} \rho(x, y) d x d y & \\
E[d]=\iint \sqrt{x^{2}+y^{2}} \rho(x, y) d x d y & \\
E[d] & =\iint r^{2} \rho(r, \theta) d r d \theta
\end{aligned}
$$

Here, $\rho(r, \theta)$ becomes constant, when the sensors are distributed uniformly in the environment.

PSO Approach: The optimization algorithm deals with the particles' velocity and position in a swarm. The movement of the particles in the swarm is referred to using the equation given. Movement of the particle to the next position from the current position is given as

$$
p^{k+1}=p^{k}+v^{k+1}
$$

The particle's velocity is given as

$$
v^{k+1}=v^{k}+x_{1} r_{1}\left(P_{p}^{k}-p^{k}\right)+x_{2} r_{2}\left(S_{p}^{k}-p^{k}\right)
$$

Where, $P_{p}^{k}$ is the particle's best position, $S_{p}^{k}$ is the swarm's best position, $\mathrm{p}$ is the particle position, $v$ is the path direction, $x_{1}, x_{2}$ are the learning coefficients commonly set to 2.0 , and $r_{1}, r_{2}$ are the random numbers with the range of $(0,1)$. 


\section{Data transmission using shortest path approach}

In this phase the data is transmitted from $\mathrm{CH}$ to $\mathrm{CH}$ till it reaches the base station. The $\mathrm{CH}$ in the communication region forwards the data to the sink since it needs high transmission power. A near optimal path is identified based on the route selection using the energy of the route. The energy of the route in the network is represented as

$$
E\left(R_{k}\right)=\sum_{i=1}^{K}\left(E_{i}^{C H}-E_{i_{-t x}}^{C H}\right)
$$

Where $E\left(R_{k}\right)$ is the energy of the route $\mathrm{k}, E_{i}^{C H}$ indicates the energy of the $\mathrm{CH}$, and $E_{i}^{C H} t_{x}$ denotes the transmission energy of the $\mathrm{CH}$. The route selection is based on inter cluster communication till the data reaches the sink. The route which has the maximum energy to carry the data to the $\mathrm{BS}$ is taken as route assignment, $R_{A}$.

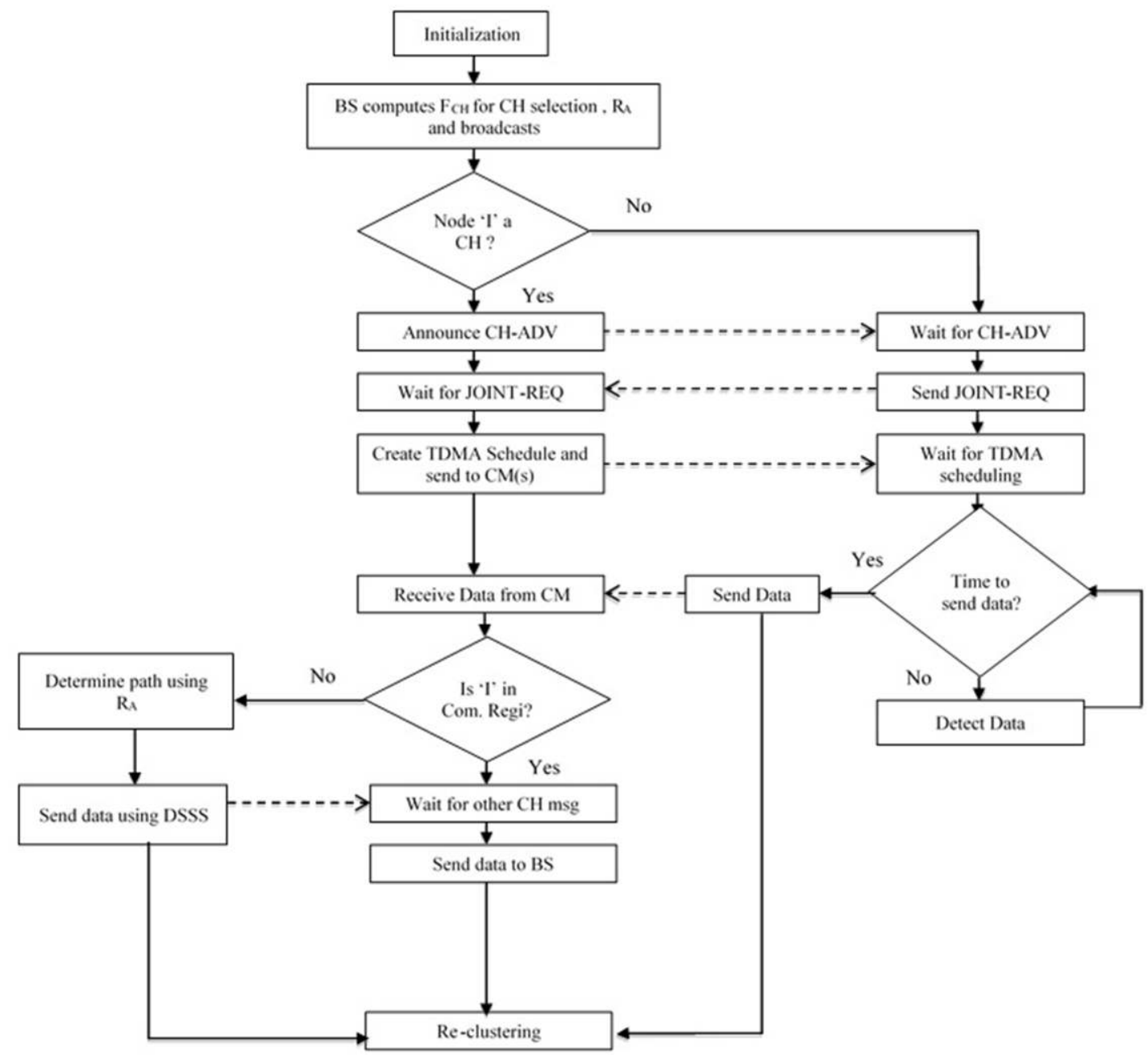

Figure 2: Flow chart of the cluster setup and data transmission

$$
R_{A}=\max \left\{E\left(R_{k}\right) ; R_{k} \epsilon P\right\}
$$


where $\mathrm{P}$ is a set of all possible routes, $\mathrm{k}$ is a $\mathrm{CH}$ that follows the route reaching the sink, and $R_{A}$ specifies the route assignment by condition. The cluster setup and data transmission flowchart is given in figure 2 .

\section{Simulation results}

The simulation was done using the math-lab simulator. The EODC performance is compared with the HHCA, HACH and LEACH-C protocol. In our simulation work, about 500 sensor nodes are placed randomly in a $500 \mathrm{~m} \times 500 \mathrm{~m}$ region. The initial energy of the node is calculated as $0.5 \mathrm{~J}$, the value of gamma is set to 0.25 and the size of the data was 500 bytes long. The simulation results are plotted in the graph and shown in figures 3 to 8 . The values of the simulation parameters for the scheme are given below,

Table 1: Simulation Parameters

\begin{tabular}{|c|c|c|}
\hline No. & Parameter & Specification \\
\hline 1 & Senor Region & $500 \times 500 \mathrm{~m}^{2}$ \\
\hline 2 & Number of sensors & 500 \\
\hline 3 & Energy of each node & $0.5 \mathrm{~J}$ \\
\hline 4 & Data size & $500 \mathrm{byte}$ \\
\hline 5 & Ec & $50 \mathrm{~nJ} / \mathrm{bit}$ \\
\hline 6 & $\epsilon_{f} s$ & $10 \mathrm{pJ} / \mathrm{bit} / \mathrm{m}^{2}$ \\
\hline 7 & $\epsilon_{m p}$ & $0.0013 \mathrm{pJ} / \mathrm{bit} / \mathrm{m}^{4}$ \\
\hline 8 & $\gamma$ & 0.25 \\
\hline 9 & Minimum Threshold energy & $10^{-4} \mathrm{~J}$ \\
\hline
\end{tabular}

The number of communication rounds increases when compared with other protocols such as LEACH-C, HHCA and HACH. The network lifespan is proportional to the number of communication rounds. The average energy dissipation at the end of 1000 rounds in EODC is $0.39 \mathrm{~nJ}$ less than $\mathrm{HHCA}$ which gives $0.48 \mathrm{~nJ}$ and in $\mathrm{HACH}$, LEACH-C the energy of the nodes drains before 1000 rounds. Figure 3 illustrates the average energy dissipation for all the nodes. The EODC performs much better than the existing protocol.

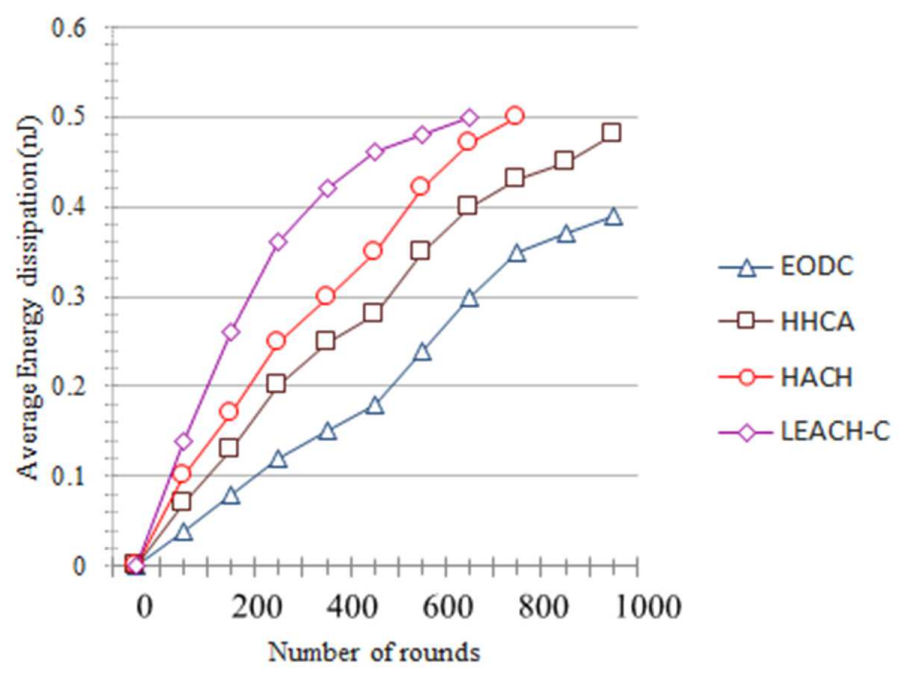

Figure 3: Comparison of average energy dissipation 
In the proposed system, the number of live nodes in the network is high when compared with the existing protocol with respect to the number of rounds. At the end of 1000 rounds in EODC, the number of alive nodes is 95; whereas in HHCA, they are 25 and in HACH, LEACH-C, the nodes are dead before 1000 rounds. Figure 4 plots the number of live nodes in each iteration.

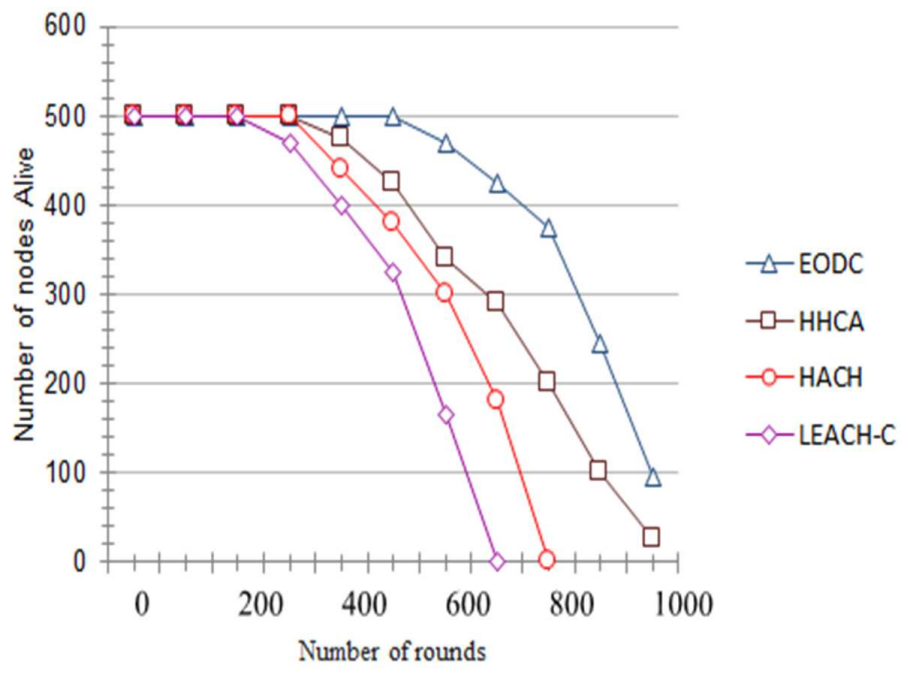

Figure 4: Number of alive nodes in each round

Figure 5 and Table 2 plot the clusters formed in the network in each round and the improvement achieved with EODC. In the proposed system, the count of clusters created at the end of 1000 rounds is 142 , which is higher when compared with the existing protocols like HHCA, HACH and LEACH- C which give 117, 92, 79 respectively. Therefore, the improvements achieved in EODC over HHCA, HACH and LEACH- C are $21.37 \%, 54.35 \%$ and $79.75 \%$ respectively.

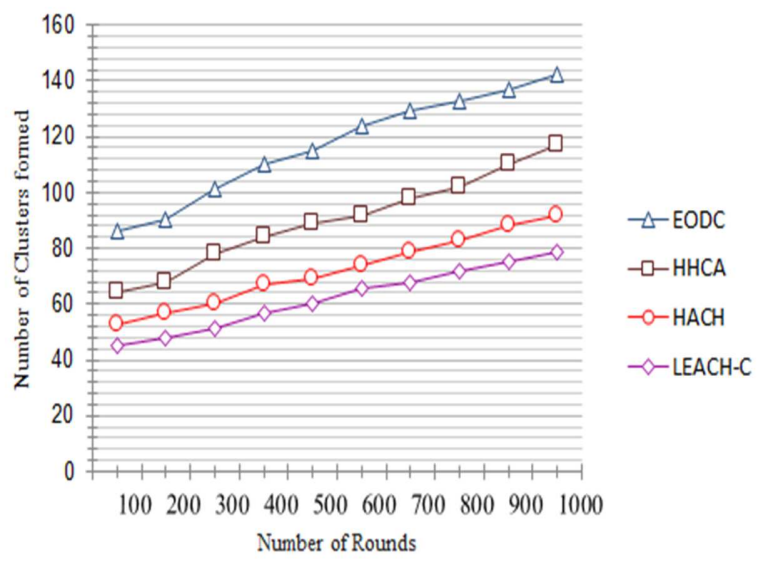

Figure 5: Clusters formed in each round 
Table 2: Cluster Formation in each round of communication

\begin{tabular}{|c|c|c|c|c|c|c|c|}
\hline \multirow{2}{*}{ Rounds } & \multicolumn{3}{|c|}{ Number of clusters formed } & \multicolumn{3}{c|}{ Improvement achieved in \% by EODC Over } \\
\cline { 2 - 8 } & EODC & HHCA & HACH & LEACH-C & HHCA & HACH & LEACH-C \\
\hline 100 & 86 & 64 & 53 & 45 & 34.38 & 62.26 & 91.11 \\
\hline 200 & 90 & 68 & 57 & 48 & 32.35 & 57.89 & 87.50 \\
\hline 300 & 101 & 78 & 60 & 51 & 29.49 & 68.33 & 98.04 \\
\hline 400 & 110 & 84 & 67 & 57 & 30.95 & 64.18 & 92.98 \\
\hline 500 & 115 & 89 & 69 & 60 & 29.21 & 66.67 & 91.67 \\
\hline 600 & 124 & 92 & 74 & 66 & 34.78 & 67.57 & 87.88 \\
\hline 700 & 129 & 98 & 79 & 68 & 31.63 & 63.29 & 89.71 \\
\hline 800 & 133 & 102 & 83 & 72 & 30.39 & 60.24 & 84.72 \\
\hline 900 & 137 & 110 & 88 & 75 & 24.55 & 55.68 & 82.67 \\
\hline 1000 & 142 & 117 & 92 & 79 & 21.37 & 54.35 & 79.75 \\
\hline
\end{tabular}

Figure 6 plots the energy dissipation of the $\mathrm{CH}$ with the given existing protocol. The energy consumed by the $\mathrm{CH}$ in EODC is $0.12 \mathrm{~nJ}$ for 200 iterations which is less than those of the existing systems like HHCA, HACH and the LEACH-C protocol which give $0.18 \mathrm{~nJ}, 0.21 \mathrm{~nJ}$ and $0.29 \mathrm{~nJ}$ respectively.

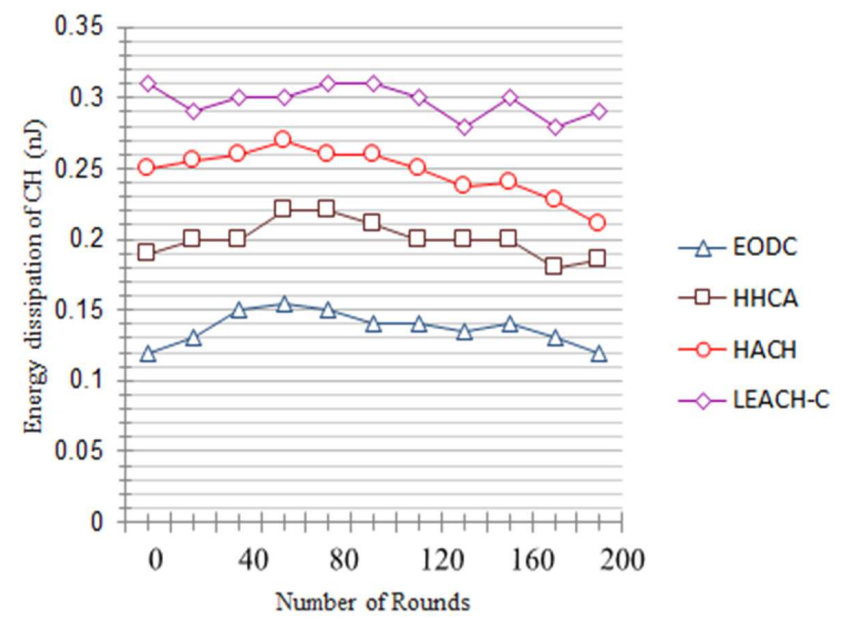

Figure 6: Energy dissipation of the $\mathrm{CH}$ under each round

Figure 7 and table 3 show the sum of residual energy during each round and the improvement achieved by EODC. In the proposed system, the residual energy of the sum of all nodes is evaluated and in comparison, the sum of network's residual energy is higher than those of the existing systems like LEACH-C, HHCA and the HACH protocol. The improvements achieved in EODC over HHCA, HACH and LEACH-C are 100\%, 211\% and $357.14 \%$ respectively. 


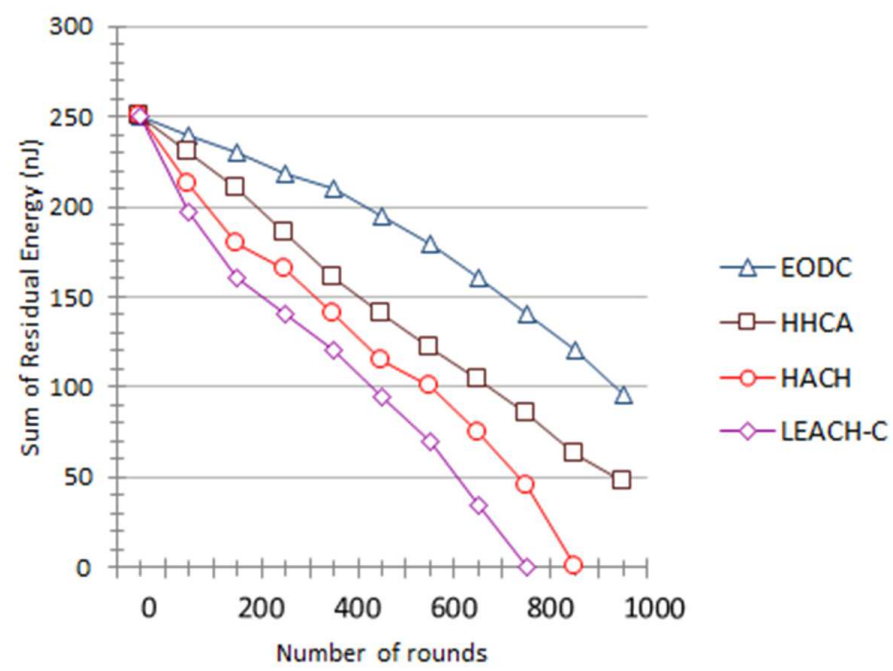

Figure 7: Sum of residual energy during each round

Table 3: Sum of Residual Energy during each round of communication

\begin{tabular}{|c|c|c|c|c|c|c|c|}
\hline \multirow{2}{*}{ Rounds } & \multicolumn{4}{|c|}{ Sum of Residual energy } & \multicolumn{3}{c|}{ Improvement achieved in \% by EODC Over } \\
\cline { 2 - 8 } & EODC & HHCA & HACH & LEACH-C & HHCA & HACH & LEACH-C \\
\hline 0 & 250 & 250 & 250 & 250 & 0.00 & 0.00 & 0.00 \\
\hline 100 & 240 & 230 & 213 & 197 & 4.35 & 12.68 & 21.83 \\
\hline 200 & 230 & 210 & 180 & 160 & 9.52 & 27.78 & 43.75 \\
\hline 300 & 218 & 185 & 165 & 140 & 17.84 & 32.12 & 55.71 \\
\hline 400 & 210 & 160 & 140 & 120 & 31.25 & 50.00 & 75.00 \\
\hline 500 & 195 & 140 & 115 & 95 & 39.29 & 69.57 & 105.26 \\
\hline 600 & 180 & 122 & 101 & 70 & 47.54 & 78.22 & 157.14 \\
\hline 700 & 160 & 104 & 75 & 35 & 53.85 & 113.33 & 357.14 \\
\hline 800 & 140 & 85 & 45 & 0 & 64.71 & 211.00 & - \\
\hline 900 & 120 & 63 & 0 & 0 & 90.48 & - & - \\
\hline 1000 & 96 & 48 & 0 & 0 & 100.00 & - & - \\
\hline
\end{tabular}

Figure 8 and table 4 plot the network lifetime comparison with LEACH-C, HHCA, and $\mathrm{HACH}$ with the EODC protocol. The lifetime gradually increases for the EODC protocol with the total number of nodes, which ranges from 250 to 500 . As per the percentage of nodes dying in every round, we calculate the lifespan of the network. The improvements achieved in EODC over HHAC, HACH and LEACH-C are $12.85 \%, 44.77 \%$ and $82.18 \%$ respectively. The proposed protocol gives better performance in comparison 


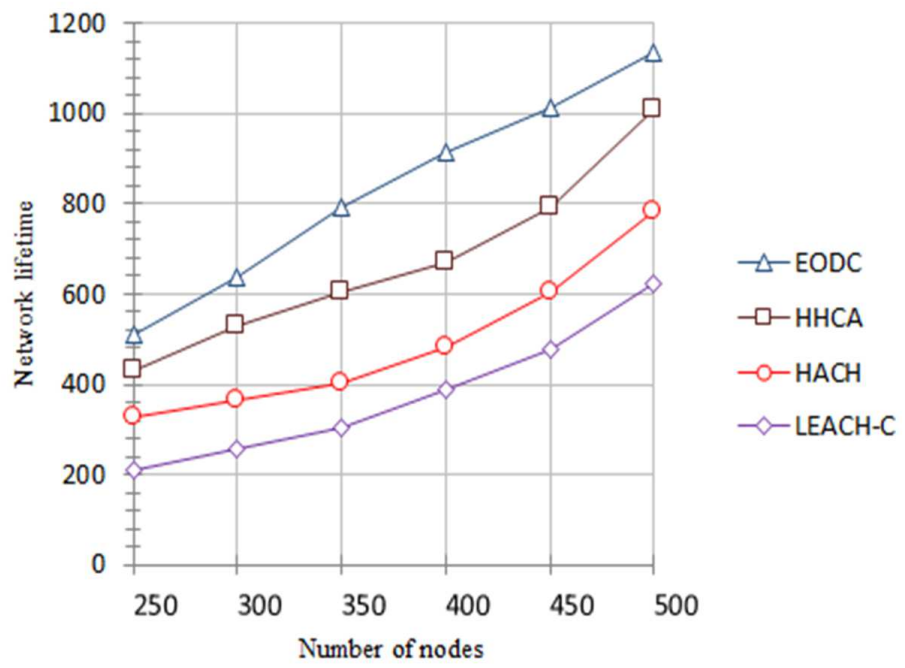

Figure 8: Lifetime Comparison of the Protocol

Table 4: Lifetime comparison of the protocols

\begin{tabular}{|c|c|c|c|c|c|c|c|}
\hline \multirow{2}{*}{$\begin{array}{c}\text { No. of } \\
\text { nodes }\end{array}$} & \multicolumn{4}{|c|}{ Lifetime Comparison } & \multicolumn{3}{|c|}{ Improvement achieved in \% by EODC Over } \\
\hline & EODC & HHCA & HACH & LEACH-C & HHCA & HACH & LEACH-C \\
\hline 250 & 510 & 431 & 328 & 212 & 18.33 & 55.49 & 140.57 \\
\hline 300 & 635 & 530 & 364 & 256 & 19.81 & 79.38 & 148.05 \\
\hline 350 & 792 & 602 & 404 & 306 & 31.56 & 96.04 & 158.82 \\
\hline 400 & 916 & 671 & 484 & 388 & 36.51 & 89.26 & 136.08 \\
\hline 450 & 1014 & 793 & 604 & 477 & 27.87 & 67.88 & 112.58 \\
\hline 500 & 1135 & 1006 & 784 & 623 & 12.85 & 44.77 & 82.18 \\
\hline
\end{tabular}

From the projected graphs of the simulation results, it is concluded that EODC has a lower energy dissipation, increase in the generation of clusters and the sum of residual energy consumed is low when compared with the preceded LEACH-C, HHCA and HACH protocols, thus increase the lifespan of the nodes and efficiency of the data transmission across the plane. The performance of EODC is compared with the above mentioned protocols with respect to energy and network lifespan. Network lifespan is characterized as the count of rounds at the point until every one of the sensor nodes comes up short on energy. It should be observed that our proposed model still has some outstanding energy beyond 1100 rounds, whereas only slight energy is remaining beyond 1000 rounds with the $\mathrm{HACH}$ protocols.

\section{Conclusion}

Energy efficiency is an essential criterion for the lifespan of the sensor network. Clustering the nodes together is the potent way to promote the energy efficiency of the network. The EODC approach is a type of hierarchical routing protocol like LEACH-C, HHCA and HACH which perform clustering of the sensor nodes. LEACH, HACH and the HACH protocol randomly choose the CHs, which in turn, increases the re-clustering process; thus more energy is being used and the network lifespan gets affected. In EODC, the cluster heads are chosen based on the PSO approach. The metrics of the proposed system are compared with the PSO model. One of 
the primary concerns is an effective $\mathrm{CH}$ selection which has been given in the proposed system. The simulation results report that in EODC, the number of clusters in the network is high in comparison. The inter cluster communication is performed using the shortest path algorithm in this scenario. The results show that by doing so the network lifetime is more in our approach when compared with the other hierarchical routing protocols. Our simulation results have proven that EODC produces better results than LEACH-C, HHCA and the HACH hierarchical routing protocol with reference to the total energy consumption and the lifetime of the network.

\section{Acknowledgments}

The authors wish to acknowledge the support extended by Department of Computer Science and Engineering, SRM Institute of Science and Technology, Chennai, India, to carry out this research work.

\section{Author contributions. Conflict of interest}

The authors contributed equally to this work. The authors declare no conflict of interest.

\section{Bibliography}

[1] Abdul Latiff, N.M.; Tsimenidis, C.C.; Sharif, B.S. (2007). Energy-Aware Clustering For Wireless Sensor Networks Using Particle Swarm Optimization, 18th Annual IEEE International Symposium on Personal, Indoor and Mobile Radio Communications, September 2007.

[2] Akyildiz, I.F.; Su, W.; Sankarasubramaniam, Y.; Cayirci, E. (2002). Wireless sensor networks: a survey, Computer Networks, 38(4), 393-422, 2002.

[3] Akkaya, A.; Younis, M. (2005). A Survey on Routing Protocols for Wireless Sensor Networks, Elsevier Journal of Ad Hoc Networks, 3(3), 325-349, 2005.

[4] Alphonse, P.J.A.; Sivaraj C.; Janakiraman T.N. (2017). An Energy-Efficient Layered Clustering Algorithm for Routing in Wireless Sensor Networks. International Journal of Distributed Systems and Technologies, 8(3), 43-66, July, 2017.

[5] Batra, P.K.; Kant, K.(2016). A clustering algorithm with reduced cluster head variations in LEACH protocol, Int. J. Syst., Control Commun, 7(4), 321-336, January 2016.

[6] Chang, C.-Y.; Chang, H.-R. (2008). Energy-aware node placement, topology control and MAC scheduling for wireless sensor networks, Comput. Netw, 52(11) , 2189-2204, August 2008.

[7] Elhabyan, R.S.; Yagoub, M.C.E. (2014). Energy Efficient Clustering Protocol for WSN using PSO, IEEE Global Information Infrastructure and Networking Symposium, September 2014.

[8] Hedetniemi, S.; Liestman, A. (1998). A Survey of Gossiping and Broadcasting in Communication Networks, Networks, 18(4), 319-349, 1998.

[9] Heinzelman, W.R.; Kulik, J.; Balakrishnan, H. (1999). Adaptive protocols for information dissemination in wireless sensor networks, 5th annual ACM/IEEE international conference on Mobile computing and networking (MobiCom '99). ACM , DOI=http://dx.doi.org/10.1145/313451.313529 
[10] Heinzelman, W.R.; Chandrakasan, A.; Balakrishnan H.(2000). Energy-efficient communication protocol for wireless microsensor networks, Proceedings of the 33rd Annual Hawaii International Conference on System Sciences, Maui, HI, USA, 2, 110, 2000.

[11] Heinzelman, W.B., Chandrakasan, A.P., Balakrishnan, H. (2002). An Application-Specific Protocol Architecture for Wireless Microsensor Networks, IEEE Transaction of Wireless Communications, 1(4), 660-670, 2002.

[12] Intanagonwiwat, C.; Govindan, R.; Estrin, D. (2000). Directed diffusion: a scalable and robust communication paradigm for sensor networks, 6th annual international conference on Mobile computing and networking (MobiCom '00). ACM, DOI=http://dx.doi.org/10.1145/345910.345920

[13] Kandris, D.; Tsioumas, P.; Tzes, A.; Nikolakopoulos. G.; Dimitrios Vergados, D.(2009). Power Conservation Through Energy Efficient Routing in Wireless Sensor Networks, Sensors, 9(9), 7320-7342, 2009.

[14] Lee, J.S.; Kao, T.Y. (2016). An Improved Three-Layer Low-Energy Adaptive Clustering Hierarchy for Wireless Sensor Networks, IEEE Internet of Things Journal, 3(6), 951-958, 2016.

[15] Li, D.; Wen, X.. (2014). An improved PSO algorithm for distributed localization in wireless sensor networks, International Conference on Software Intelligence Technologies and Applications 83 International Conference on Frontiers of Internet of Things 2014, Hsinchu, 184-189, 2014.

[16] Lindsey, S.; Raghavendra, C. (2002). Data Gathering Algorithm in Sensor Networks Using Energy Metrics, IEEE Transactions on Parallel and Distributed Systems, 13(9), 924-935, 2002.

[17] Logambigai, R.; Kannan, A. (2018). Energy conservation routing algorithm for wireless sensor networks using hybrid optimisation approach, International Journal of Communication Networks and Distributed Systems, 20(3), 352-371, January, 2018.

[18] Lotf, J.; Bonab, M.; Khorsandi, S. (2006). A Novel Cluster-Based Routing Protocol with Extending Lifetime for Wireless Sensor Networks, 5th International Conference on Wireless and Optical Communications Networks, East Java Indonesia, Surabaya, 1-5, 2006.

[19] Mao, J.; Wu, Z.; Wu, X. (2007). A TDMA scheduling scheme for many-to-one communications in wireless sensor networks, Computer Communication, 30(4), 863-872, 2007.

[20] Muruganathan, D.; Bhasin, R. (2005). A Centralized Energy Efficient Routing Protocol for Wireless Sensor Networks, IEEE Communication Magazine, 43(3), 8-13, 2005.

[21] Oladimejia, M.O.; Turkeya, M.; Dudleya, S. (2017). HACH: Heuristic Algorithm for Clustering Hierarchy Protocol in Wireless Sensor Network, Applied Soft Computing, 452-460, 2017.

[22] Rao, P. C.; Jana, Prasanta K.; Haider Banka. (2017). A particle swarm optimization based energy efficient cluster head selection algorithm for wireless sensor networks, Wireless Networks, 23(7), 2005-2020, 2017.

[23] Sangho Yi; Junyoung Heo; Yookun Cho; Jiman Hong.(2007). PEACH: Power-efficient and adaptive clustering hierarchy protocol for wireless sensor networks, Comput. Commun, 30, 2842-2852, 2007. 
[24] Sarkar, A.; Senthil Murugan, T. (2019). Cluster head selection for energy efficient and delay-less routing in wireless sensor network. Wireless Network, 25(1), 303-320, 2019.

[25] Thein, M.C.M.; Thein, T. (2010). An energy efficient cluster head selection for wireless sensor networks, Proceedings of the UKSim/AMSS 1st International Conference on Intelligent Systems, Modelling and Simulation, 287-291, 2010.

[26] Wu, Y.; Fahmy, S.; Shroff, N.(2007). Energy Efficient Sleep/Wake Scheduling for MultiHop Sensor Networks: non-Convexity and Approximation Algorithm, 26th Annual IEEE Conference on Computer Communications, Anchorage, Alaska, 1568-1576, 2007.

[27] Younis, O.; Fahmy, S. (2004). HEED: A Hybrid, Energy-Efficient Distributed Clustering Approach for Ad Hoc Sensor Networks, IEEE Transactions on Mobile Computing, 3(4), 366-379, 2004.

[28] Zhao, S.; Wu, J.; Jiezhang; LiefengLiu; kaiyun Tian.(2014). A General Self-Organized TreeBased Energy-Balance Routing Protocol for Wireless Sensor Network, IEEE Transactions on Nuclear Science, 61(2), 732- 740, 2014.

[29] Zhang, D.; Li, G.; Zheng, K.; Ming, X.; Pan, Z.H.(2014). An Energy Balanced Routing Method Based on Forward-Aware Factor for Wireless Sensor Networks, IEEE Transactions on Industrial Informatics, 10(1), 766-773, 2014.

[30] Zhou, Y.; Wang, N.; Xiang, W. (2016). Clustering Hierarchy Protocol in Wireless Sensor Networks Using an Improved PSO Algorithm, IEEE Access, 5, 2241-2253, 2016. 